\title{
Sarcopenia, But Not Sarcopenic Obesity, is Associated with Clinically Significant Depressive Symptoms in Older Mexican Adults
}

\author{
Adrián Martínez-Ruiz , Paloma Roa-Rojas² and Oscar Rosas-Carrasco 2* \\ ${ }^{1}$ Demographics Epidemiology and Social Determinants Department, National Institute of Geriatrics of Mexico, Mexico \\ ${ }^{2}$ Clinical Epidemiology Department, National Institute of Geriatrics of Mexico, Mexico
}

${ }^{*}$ Corresponding author: Oscar Rosas-Carrasco, Clinical Epidemiology Department, National Institute of Ge riatrics of Mexico, Mexico, Te I: + 52 52782348; E-mail: oscar_rosas_c@hotmail.com

Received date: June 9, 2018; Accepted date: June 29, 2018; Published date: July 3, 2018

Copyright: (C) 2018 Martínez-Ruiz A, et al. This is an open-access article distributed under the terms of the Creative Commons Attribution License, which permits unrestricted use, distribution, and reproduction in any medium, provided the original author and source are credited.

Citation: Rosas-Carrasco O, Roa-Rojas P, Martínez-Ruiz A (2018) Sarcopenia, but not sarcopenic obesity, is associated with clinically significant depressive symptoms in older Mexican adults. Clin Psychiatry Vol.4 No.2:5.

\section{Abstract}

Background: Sarcopenia and obesity have a high prevalence in Mexico. Evidence suggests that there is an association between these disorders and clinically significant depressive symptoms.

Objective: The aim of the study is to provide the rate and association of depressive symptoms in subjects with sarcopenia and sarcopenic obesity in elderly Mexican.

Methods: This study is a cross-sectional analysis of data obtained from the FraDySMex study conducted in Mexico City $(n=513)$. Depressive symptoms were assessed with the short version of the Center for Epidemiological StudiesDepression scale, and a cut-off point $\geq 5$ indicated the presence of clinically significant depressive symptoms. A body mass index $\geq 30$ defined obesity. Sarcopenia was assessed with the SARC-F questionnaire; a score $\geq 4$ indicated the presence of sarcopenia. Sarcopenic obesity was defined as obesity along with sarcopenia. Two models were used to determine the association: one model assessed sarcopenia and depressive symptoms; the other one assessed them with sarcopenic obesity.

Results: The analysis included 513 subjects, and the mean age of the subjects was $71.7 \pm 9.4 ; 80.1 \%$ were female. The prevalence of CSDS, sarcopenia and sarcopenic obesity was $40.5 \%, 10 \%$ and $7.9 \%$, respectively. Sarcopenia was associated with depressive symptoms (OR 2.64, 95\% Cl 1.35-5.14, $p=0.004)$, but sarcopenic obesity was not associated with them (OR 1.86, 95\% $\mathrm{Cl} 0.89-3.89, \mathrm{p}=0.09$ ).

Conclusion: Our study found that subjects with only sarcopenia were more likely to have depressive symptoms than subjects with sarcopenic obesity. However, properly prospective studies are needed to better understand this association.

Keywords: Sarcopenia; Sarcopenic obesity; Depression

\section{Introduction}

As in other developing countries, the Mexican population is aging. This phenomenon poses urgent concerns for many fields related to public health. In terms of mental health issues, geriatric depression has a prevalence of $1.3 \%$ to $5.1 \%$ [1], and the prevalence for clinically significant depressive symptoms (CSDS) in older Mexican adults is up to $29.1 \%$ [2].

There is growing evidence of an association between depression, obesity, and sarcopenia. Currently, obesity is a major health issue in the Mexican population. The prevalence of obesity per the body mass index (BMI) is $32.4 \%$, and the prevalence of obesity per abdominal adiposity is 74\%; moreover, the prevalence of these factors has highly increased annually over the past decade [3]. The association between obesity and depression is well known [4]. Longitudinal studies have shown that obesity is a risk factor for the onset of depression and that depression increases the odds of developing obesity [5]. Furthermore, this association is stronger for women than for men [6].

Likewise, sarcopenia in older Mexican adults has a prevalence of $9.3-20.8 \%$ [7]. The data indicate a relationship between depressive symptoms and sarcopenia. A recent study performed by Remigio-Baker and colleagues [8] found that a low lean muscle mass for locomotion predicts elevated depressive symptoms in men.

The evidence also suggests that there is a synergistic impact of sarcopenia and obesity (sarcopenic obesity) on the risk of depressive symptoms. A study conducted with older Japanese adults found that depressive symptoms were more strongly associated with sarcopenic obesity than in older adults without sarcopenic obesity [9]. Another study [10] found an association between an adverse psychological health status, such as depressive symptoms and suicidal ideation, and sarcopenic obesity. These findings were confirmed in other studies [11,12]. Furthermore, this synergistic effect has also been confirmed in longitudinal cohort studies. Hamer and colleagues found that after a six-year follow-up time, the onset of new depressive 
symptoms was associated with the presence of sarcopenic obesity [13].

Nevertheless, the association between sarcopenia or obesity alone and depressive symptoms is inconclusive. Moreover, evidence exists that suggests that sarcopenia and obesity are not associated with depressive symptoms. For example, a study conducted in older Korean adults did not find an association between sarcopenia, sarcopenic obesity or obesity and depressive symptoms [14].

Other studies have found an association between sarcopenia and cognitive impairment or depressive symptoms [15]. In a study conducted with Taiwanese men, an association between cognitive impairment, depression and sarcopenia was observed.

The major diagnostic feature of sarcopenia was defined by the loss of muscle mass. However, current definitions from the European Working Group on Sarcopenia in Older People (EWGSOP), the International Working Group on Sarcopenia (IWGS) and the Asian Working Group for Sarcopenia (AWGS), pose that the diagnostic criteria of the sarcopenic syndrome must contain both low muscle mass and impaired muscle function (defined either by muscle strength or physical performance). However, muscle mass and function measurement can be time-consuming and requires specialized equipment, which increases its cost significantly. Hence, current diagnostic methods of sarcopenia can be separated into methods based on objective measures such as dual-energy $X$-ray absorptiometry, the studies previously cited used this type of methods, and diagnostic methods based on information provided by the subjects such as the SARC-F questionnaire.

The SARC-F questionnaire is a simple and rapid screening instrument for sarcopenia, already validated in several countries, including Mexico. It has shown it can predict physical limitations comparable to the consensus panels EWGSOP, IWGS, and AWGS. Among its many advantages, the sarcopenia diagnosis can be made by minimally trained health personnel and it can be used for large-scale epidemiological studies.

Considering that obesity and sarcopenia have a high prevalence in Mexican populations, a study of the association between these diseases and depressive symptoms is needed. The aim of this study was to analyse the associations between sarcopenia, sarcopenic obesity and depressive symptoms in older Mexican adults.

\section{Materials and Methodology}

\section{Subjects}

The current study is a cross-sectional analysis of data obtained in the FraDySMex (Frailty Dynapenia and Sarcopenia in Mexican Adults) cohort study. Details of the study design and participant selection can be found elsewhere [16]. Briefly, the FraDySMex study was a community-based cohort study conducted in Mexico City. The subjects were invited to participate in the study from October 2014 to December 2015. Psychologist or social workers visited them in their homes or in elderly community centers and social security centers. Also, flayers were left outside of churches and health centers. All individuals included in this study were ambulatory, with or without assisting devices and were able to answer the study questionnaire. If subjects obtained a Mini-Mental State Examination (MMSE) score of 10 points or less, the study questionnaire was answered with the caregivers help. The study included individuals aged 50 years and older and was structured into two phases: October 2014 to December 2014 was the first phase; and October 2015 to December 2015 was the second phase. Each phase included a series of objective geriatric and psychological evaluations. All evaluations were conducted by trained staff, at the Functional Evaluation Research Laboratory at the National Institute of Geriatrics of Mexico (INGer). Subjects who were institutionalized, had any kind of decreased alertness or an acute condition that compromised the ability to answer the questionnaire, were excluded. Written Informed consent was obtained from all participants prior to the study. The current study is a cross-sectional analysis of data collected in the first phase of the FraDySMex study. The subjects included in this analysis had to have the complete data required to conduct this analysis. All subjects were classified into two groups based on the presence of CSDS. The FraDySMex study was approved by the institutional review boards of participant institutions (the Angeles Mocel General Hospital and INGer, DI-PI-002/2014).

\section{Measurements}

Sociodemographic characteristics: The sociodemographic variables evaluated were as follows: age ( $>50$ years old); sex (female versus male); and level of education, which was divided into basic (primary and secondary) education and middle and professional studies (higher education, high school, undergraduate and postgraduate degrees). These categories corresponded to the Mexican education system. Civil status was categorized into a married or de facto relationship and other.

Clinical features: To evaluate the presence of CSDS a short version of the depression scale of the Center for Epidemiologic Studies (CES-D 7), which has been validated in a Mexican elderly population [17], was used. The CES-D 7 includes seven questions that evaluate five symptoms: three questions for dysphoric mood and one question each for motivation, concentration, loss of pleasure and poor sleep. A cut-off point of 5 or more indicated the presence of CSDS [17].

Obesity was classified based on the World Health Organization criteria using the BMI. The BMI was calculated by dividing the body weight by height squared $\left(\mathrm{kg} / \mathrm{m}^{2}\right)$. Individuals with a BMI of 30 or more were considered obese [18]

Sarcopenia was assessed with the Mexican version of the SARC-F questionnaire. The SARC-F questionnaire includes 5 components: strength, walking assistance, sit-to-stand performance, stair climbing and falls. Each question is scored from 0 (no difficulty or 0 falls) to 2 (a lot of difficulty and 4 or more falls). A total score of 4 or more indicates the presence of sarcopenia [7]. Sarcopenic obesity was defined as the presence of obesity with accompanying loss of muscle mass and strength.

It has been reported that the SARC-F reliability (Cronbach alpha of 0.641 ), sensibility, specificity, positive an d negative 
predictive values are adequate for the sarcopenia diagnosis [7] Furthermore, high specificity for SARC-F has also been reported in Mexican population (specificity compared to the European Working Group on Sarcopenia in Older People $=82.2 \%$, the International Working Group on Sarcopenia $=83.3 \%$ and the Asian Working Group for Sarcopenia=82.1\%). Also, statistically significant correlations between the SARC-F total score and other measures related to sarcopenia like age, quality of life, self-rated health status, cognition, dependence in activities of daily living, nutritional status, depression, gait speed, grip strength, peak torque and power for knee extension, SPPB, balance, SMI, and frailty were found [7].

A validated version of the MMSE in the Mexican elderly population was used to assess cognitive status. A cut-off point of 24 was used to classify the subjects with or without cognitive impairment [19].

To evaluate the presence of comorbidity, we used the Spanish version of the Charlson comorbidity index (CCI) [20]. The $\mathrm{CCl}$ assesses the presence of 19 diseases, two of which are assessed with and without complications (diabetes and liver disease). The information of the $\mathrm{CCl}$ was collected during direct interviews with the individuals or caregiver. For this study, the total score of the $\mathrm{CCl}$ was calculated, and a cut-off point of 4 or more was used to classify the results [21]. The presence of gastritis, diabetes with and without complications, and cerebrovascular disease was concluded directly from the $\mathrm{CCl}[20]$.

Physical performance was assessed with the Short Physical Performance Battery (SPPB) score, which combines the evaluation of walking speed, sit-to-stand performance and standing balance. The scores range from 0 (being the worst performance) to 12 (being the best performance). A cut-off point of 9 was used to classify the individuals. All tests were evaluated by qualified medical staff and according to the SPPB instructions. Walking speed was evaluated during a 6-meter walk in the GAIT Rite (platinum 20) instrumented walkway (204 $\times 35.5 \times 0.25$ inches, sample rate $100 \mathrm{~Hz}$ ), positioned along a straight section of the walkway [22].

Independence was measured with the Barthel Index of Activities of Daily Living tool. This assessment evaluates feeding ability, wheelchair transfers, toilet use, mobility on and off the toilet, bathing, mobility on a level surface, mobility up and down stairs, dressing, bowel control and bladder control. The scores of the index ranges from 0-100 (0 means completely dependent and 100 means completely independent). A cut-off point of $\geq 95$ was used to indicate independence [23].

Polypharmacy refers to the ingestion of many medications and can represent unnecessary medication use. Subjects who ingested 5 or more medications were classified as subjects with polypharmacy [24].

Statistical analysis: Data were analysed using PASW statistics version 21 (SPSS, Chicago, IL, USA). Descriptive statistics were reported as the means \pm standard deviation for continuous variables and as frequencies and percentages for categorical variables. Differences in sociodemographic and clinical features between the groups with and without CSDS were examined using Student's t-test for continuous variables a nd t he chisquare test for categorical variables.

To evaluate the association between sarcopenia and sarcopenic obesity with CSDS, odds ratios with $95 \%$ confidence intervals ( $\mathrm{Cls}$ ) were obtained by conducting a logistic regression analysis. Two final adjusted models are presented. The first model evaluated the association of sarcopenia with CSDS, and the second model evaluated the association of sarcopenic obesity with CSDS. Significant variables for the final models were first selected by a forward selection method and only the variables that showed statistical significance from the initial logistic regression analysis were selected and included in the final models. The level of statistical significance was set at $5 \%$.

\section{Results}

A total of 513 individuals were included in the study. The mean age of the study population was 71.7 (SD \pm 9.4 ) years. A total of $37.2 \%(n=191)$ were married, $48.8 \%$ had a basic education level, and $80.1 \%(n=411)$ were women with an average age of 71.7 (SD \pm 9.4 ). The prevalence of CSDS for the whole sample was $40.5 \%$ (Table 1 ). The sociodemographic and clinical characteristics according to the presence of CSDS are shown in Table 2. The prevalence of sarcopenia was $10 \%$, and the prevalence of sarcopenic obesity was $7.9 \%$. Compared to males, females showed a higher rate of CSDS (44.5\% vs. $24.5 \%$, respectively, $p \leq 0.001$ ), and individuals with lower levels of education showed higher rates of CSDS (49.8 vs. 31.8, $p \leq 0.001$ ). There was no statistically significant difference in age and marital status. Concerning clinical variables, statistically significant differences for comorbidity $(p=0.04)$, acid peptic disorder $(p \leq 0.001)$, diabetes with complications $(p=0.009)$, low physical performance (SPPB) $(p=0.04)$ and cognitive impairment ( $p \leq 0.001$ ) were found. Similarly, the presence of sarcopenia $(p=0.000)$ and sarcopenic obesity $(p=0.005)$ showed a statistically significant difference between subjects with CSDS and subjects without CSDS. However, there were no significant differences for obesity $(p=0.51)$.

The logistic regression analysis for CSDS according to the presence of sarcopenia and sarcopenic obesity are shown in Table 3. These models are adjusted per significant sociodemographic variables (gender and education) and the other variables that showed statistical significance (comorbidity, gastritis, diabetes with complications, low physical performance and cognitive impairment). The sarcopenic analysis model showed that men had a lower risk of presenting with CSDS than women (OR 0.4; 95\% Cl 0.29-0.83). The model also showed that individuals with higher education had less risk of presenting with CSDS (OR 0.5; 95\% Cl 0.38-0.87). Subjects with low physical performance and diabetes with complications showed a significative risk of presenting with CSDS (OR 1.08; 95\% Cl 0.711.64 and $\mathrm{OR} 2.4 ; 95 \% \mathrm{Cl} 1.08-5.42$, respectively). Additionally, the presence of sarcopenia was associated with having CSDS (OR 2.64; $95 \% \mathrm{Cl}$ 1.35-5.14). Meanwhile, the sarcopenic obesity model (Table 3), did not found a significant association between 
sarcopenic obesity and the presence of CSDS (OR 1.86; $95 \% \mathrm{Cl}$ 0.89-3.89).

Table 1. Demographic and clinical characteristics of the studied population.

\begin{tabular}{|c|c|}
\hline Variables & Mean (SD) \\
\hline Age (years) & $71.7( \pm 9.4)$ \\
\hline Education & $\mathrm{n}(\%)$ \\
\hline Basic education & $249(48.8)$ \\
\hline Higher education & $261(51.1)$ \\
\hline \multicolumn{2}{|l|}{ Marital status } \\
\hline Married/civil union/de facto & $191(37.2)$ \\
\hline Other & $319(62.2)$ \\
\hline \multicolumn{2}{|l|}{ Gender } \\
\hline Female & $411(80.1)$ \\
\hline Male & $102(19.9)$ \\
\hline Clinical significant depressive symptoms (CES-D 7 scale $\geq 5$ ) & $208(40.5)$ \\
\hline Comorbidity (Charlson index $\geq 4$ ) & $63(12.2)$ \\
\hline Acid peptic disorder (yes) & $205(39.9)$ \\
\hline Diabetes without complications & $100(19.5)$ \\
\hline Diabetes with complications (yes) & $34(6.6)$ \\
\hline Cerebrovascular disease (yes) & $11(2.1)$ \\
\hline Physical performance (SPPB total score $\leq 9$ ) & $237(46.4)$ \\
\hline Cognitive impairment (MMSE<24) & $67(13.1)$ \\
\hline Dependency (Barthel index $\leq 95$ ) & $105(20.5)$ \\
\hline Polypharmacy ( $\geq 5$ medications) & $221(43.1)$ \\
\hline Obesity alone group & $116(22.6)$ \\
\hline Sarcopenia alone group (SARC-F $\geq 4$ ) & $48(10.0)$ \\
\hline Sarcopenic obesity group & $37(7.96)$ \\
\hline \multicolumn{2}{|c|}{$\begin{array}{l}\text { The means and standard deviations are shown for continuous variables. } \\
\text { Proportions are shown as percentages for categorical variables. Abbreviations: } \\
\text { SD: standard deviation, CES-D: Center for Epidemiological Studies-Depression } \\
\text { Scale, MMSE: Mini-mental State Examination, SPPB: Short Physical } \\
\text { Performance Battery. }\end{array}$} \\
\hline
\end{tabular}

Table 2. Comparison of demographic and clinical characteristics of the study participants with and without depressive symptoms.

\begin{tabular}{|c|c|c|c|}
\hline Age & $71.46( \pm 8.94)$ & $72.1( \pm 10.1)$ & 0.07 \\
\hline \multicolumn{4}{|l|}{ Education } \\
\hline Basic education & $125(41.3)$ & $124(59.9)$ & \multirow{2}{*}{0.00} \\
\hline High/professional education & $178(58.7)$ & $83(40.1)$ & \\
\hline \multicolumn{4}{|l|}{ Marital status } \\
\hline Married/civil union/de facto & $117(38.6)$ & $74(35.7)$ & \multirow{2}{*}{0.51} \\
\hline Other & $186(61.4)$ & $133(64.3)$ & \\
\hline \multicolumn{4}{|l|}{ Gender } \\
\hline Female & $228(74.8)$ & $183(88.0)$ & \multirow{2}{*}{$\begin{array}{l}0.00 \\
0\end{array}$} \\
\hline Male & $77(25.2)$ & $25(12.0)$ & \\
\hline $\begin{array}{l}\text { Comorbidity (Charlson index } \\
\geq 4 \text { ) }\end{array}$ & $30(9.8)$ & $33(15.9)$ & 0.04 \\
\hline Acid peptic disorder (yes) & $97(31.8)$ & $108(51.9)$ & $\begin{array}{l}0.00 \\
0\end{array}$ \\
\hline $\begin{array}{ll}\text { Diabetes } & \text { without } \\
\text { complications } & \end{array}$ & $60(19.7)$ & $40(19.2)$ & 0.90 \\
\hline $\begin{array}{l}\text { Diabetes with complications } \\
\text { (yes) }\end{array}$ & $13(4.3)$ & $21(10.1)$ & $\begin{array}{l}0.00 \\
9\end{array}$ \\
\hline $\begin{array}{l}\text { Cerebrovascular disease } \\
\text { (yes) }\end{array}$ & $5(1.6)$ & $6(2.9)$ & 0.33 \\
\hline SPPB battery total score $\leq 9$ & $152(50.2)$ & $85(41.1)$ & 0.04 \\
\hline $\begin{array}{ll}\text { Cognitive } & \text { impairment } \\
(\mathrm{MMSE}<24) & \end{array}$ & $30(6.6)$ & $37(12.5)$ & $\begin{array}{l}0.00 \\
9\end{array}$ \\
\hline $\begin{array}{l}\text { Dependency (Barthel index } \leq \\
\text { 95) }\end{array}$ & $54(17.7)$ & $51(24.5)$ & 0.06 \\
\hline $\begin{array}{l}\text { Polypharmacy } \\
\text { medications })\end{array} \quad(\geq \quad 5$ & $131(43.2)$ & $90(43.5)$ & 0.95 \\
\hline Obesity alone & $72(23.6)$ & $44(21.2)$ & 0.51 \\
\hline Sarcopenia alone & $18(5.9)$ & $30(14.4)$ & $\begin{array}{l}0.00 \\
0\end{array}$ \\
\hline Sarcopenic obesity & $15(4.9)$ & $22(10.6)$ & $\begin{array}{l}0.00 \\
5\end{array}$ \\
\hline \multicolumn{4}{|c|}{$\begin{array}{l}\text { The means and standard deviation are shown for continuous variables. } \\
\text { Proportions are shown as percentages for categorical variables. P values for } \\
\text { group differences were calculated with Student's t-test for continuous variables } \\
\text { and with the chi-squared test for categorical variables. Abbreviations: MMSE: } \\
\text { Mini-mental State Examination, SPPB: Short Physical Performance Battery. }\end{array}$} \\
\hline
\end{tabular}

\begin{tabular}{|l|l|l|l|}
\hline & Without & With & P \\
depressive & depressive & valu \\
& symptoms & symptoms & e \\
& $n=305(59.5 \%)$ & $n=208(40.5 \%)$ & \\
\hline
\end{tabular}

Table 3. Unadjusted and Adjusted Models for Sarcopenia, Sarcopenic Obesity and other factors associated with CSDS.

\begin{tabular}{|l|l|l|l|}
\hline & Unadjusted model & Model 1 & Model 2 \\
\hline
\end{tabular}




\begin{tabular}{|c|c|c|c|c|c|c|c|c|c|}
\hline & & & & \multicolumn{3}{|c|}{ Adjusted Model for Sarcopenia } & \multicolumn{3}{|c|}{ Adjusted Model for SO } \\
\hline & OR & $95 \% \mathrm{Cl}$ & $\begin{array}{l}P \\
\text { value }\end{array}$ & OR & $95 \% \mathrm{Cl}$ & $\begin{array}{l}P \\
\text { value }\end{array}$ & OR & $95 \% \mathrm{Cl}$ & $\begin{array}{l}P \\
\text { value }\end{array}$ \\
\hline Age (years) & 1.00 & $0.97-1.02$ & 0.80 & - & - & - & & - & - \\
\hline Female (vs. male) & 0.40 & $0.24-0.66$ & 0.000 & 0.49 & $0.29-0.83$ & 0.009 & 0.55 & $0.32-0.96$ & 0.03 \\
\hline Higher education (vs. basic education) & 0.47 & $0.32-0.67$ & 0.000 & 0.57 & $0.38-0.87$ & 0.009 & 0.51 & $0.34-0.77$ & 0.002 \\
\hline Married (vs. not married) & 0.88 & $0.61-1.23$ & 0.51 & - & - & - & - & - & - \\
\hline High comorbidity (vs. low comorbidity) & 1.72 & $1.01-2.93$ & 0.04 & 1.48 & $0.79-2.80$ & 0.21 & 1.18 & $0.62-2.25$ & 0.59 \\
\hline Acid peptic disorder (vs. no) & 2.31 & $1.61-3.33$ & 0.000 & 1.83 & $1.21-2.74$ & 0.004 & 1.99 & $1.32-3.00$ & 0.001 \\
\hline Diabetes without complications (vs. no) & 0.97 & $0.62-1.51$ & 0.90 & - & - & - & - & - & - \\
\hline Diabetes with complications (vs. no) & 2.52 & $1.23-5.16$ & 0.01 & 2.42 & $1.08-5.42$ & 0.03 & 2.42 & $1.02-5.71$ & 0.04 \\
\hline Cerebrovascular disease (vs. no) & 1.48 & $0.35-6.28$ & 0.59 & - & - & - & - & - & - \\
\hline Poor physical performance (vs. no) & 0.69 & $0.48-0.98$ & 0.04 & 1.08 & $0.71-1.64$ & 0.71 & 0.96 & $0.63-1.46$ & 0.85 \\
\hline Cognitive impairment (vs. no) & 2.03 & $1.10-3.75$ & 0.02 & 1.71 & $0.94-3.10$ & 0.07 & 1.49 & $0.85-2.71$ & 0.18 \\
\hline Dependent (vs. independent) & 0.93 & $0.54-1.58$ & 0.79 & - & - & - & - & - & - \\
\hline Polypharmacy (vs. no) & 0.74 & $0.47-1.16$ & 0.19 & - & - & - & - & - & - \\
\hline $\begin{array}{l}\text { Sarcopenia } \\
\text { (vs. without sarcopenia) }\end{array}$ & 2.90 & $1.56-5.38$ & 0.001 & 2.64 & $1.35-5.14$ & 0.004 & - & - & - \\
\hline \multirow[t]{2}{*}{$\begin{array}{l}\text { Sarcopenic obesity } \\
\text { (vs. without sarcopenic obesity) }\end{array}$} & 1.87 & $0.89-3.95$ & 0.09 & - & - & - & 1.86 & $0.89-3.89$ & 0.09 \\
\hline & \multicolumn{3}{|c|}{ Hosmer \& Lemeshow $=0.90$} & \multicolumn{3}{|c|}{ Hosmer \& Lemeshow=0.91 } & \multicolumn{3}{|c|}{ Hosmer \& Lemeshow= 0.89} \\
\hline
\end{tabular}

\section{Discussion}

In the current study, our main goal was to analyse the relationship between sarcopenia, sarcopenic obesity and CSDS symptoms in older Mexican adults. We expected CSDS to be highly prevalent, and we hypothesized that CSDS would be related to sarcopenia and sarcopenic obesity. However, we found an association between CSDS and sarcopenia but not between CSDS and sarcopenic obesity.

The association between depression and sarcopenia has been previously reported. The study conducted by Remigio-Baker and colleagues [8] showed that according to the scores from the CESD scale, the depressive symptoms of Chinese men were associated with lower muscle mass, particularly in the muscles used for locomotion. These findings were similar to the results of an English longitudinal study that included 3862 community dwelling participants. The authors also found an association between sarcopenia and the onset of depressive symptoms. These findings were particularly marked in the obese subjects at the study baseline [13].

Two main hypotheses have been proposed to explain these findings. The first states that the muscle mass system is implicated in the mental health outcome of wellbeing through adequate glucose regulation [9]. The second hypothesis states that an elevated presence of cortisol is related to increases in inflammatory markers that may promote decreased muscle mass. Therefore, both hypotheses suggest a pathological cycle between the loss of muscular mass and the presence of depressive symptoms. However, the data remains inconclusive.

Regarding sarcopenic obesity and its association with CSDS, we predicted that we would find a statistically significant association between sarcopenic obesity and CSDS, we did not find such an association. There are three possible explanations for our findings. First, the association between sarcopenic obesity and depressive symptoms may not be synergistic. Second, obesity alone is not necessarily associated with depression. Third, evidence suggests that the Mexican population have a misperception of BMI.

Regarding the first explanation, evidence of the association between sarcopenic obesity and the presence of CSDS is inconclusive. For example, Ishii and colleagues [9] suggest that sarcopenic obesity, but not sarcopenia or obesity alone, is associated with depressive symptoms. Additionally, in a longitudinal study, Hamer and colleagues [13] found that depressive symptoms were associated with sarcopenic obesity. However, another study found positive associations between self-stress perception and sarcopenic obesity and between suicidal ideation and sarcopenic obesity; there was no association between CSDS and sarcopenic obesity. 
Because other studies have shown that obesity alone is not necessarily associated with depression, our second explanation may partially explain our findings. For example, a 16-year followup cohort French study reported that depressive symptoms were associated with obesity if the subjects also had poor metabolic health (evaluated through cardiovascular risk factors) [25]. Although the mechanisms that explain the association between obesity and CSDS remain unclear.

The "jolly fat" hypothesis states the opposite association between obesity and CSDS. A Brazilian study showed that subjects with severe obesity had a similar incidence of depressive symptoms to that of subjects with a normal BMI during a 4-year follow-up period. However, the prevalence of CSDS was higher in the subjects with severe obesity [26]. Moreover, Qian and colleagues [27] found an inverse association between obesity and depressive symptoms. They suggested a partial explanation: obese people do not have strict diets and are therefore less likely to present depressive symptoms.

Third, our findings can also be explained by misperception of BMI. Some studies show that underestimating or overestimating BMI can be associated with the presence or absence of depressive symptoms [28]. Heard and colleagues [29] found that underestimating weight was associated with a higher quality of life. Considering that Mexico has one of the highest rates of obesity in the world, underestimating a person's weight could explain our results [30]. A study conducted with 32050 Mexican subjects reported that up to $58 \%$ of the subjects underestimated their BMI [31]. Thus, underestimation of weight can lead to a lower frequency of depressive symptoms.

However, since obesity is a complex and multifactorial disease, it is important to consider the possibility that its association with other diseases such as depression may be different depending on the context. If we want to clarify the association between obesity and depression, more studies that consider it as a multifactorial phenomenon are needed.

This study has several limitations that should be acknowledged. First, due to the nature of cross-sectional studies, the temporality of the phenomena cannot be considered for analysis. Second, the sample size is limited and cannot be generalized to the older population as a whole. Third, the SARC-F questionnaire is a valid instrument for diagnosing sarcopenia, however, the diagnosis is based on information provided by the subjects rather than on objective measures such as dual-energy X-ray absorptiometry. Therefore, properly conducted prospective studies with larger samples are needed for a better understanding of this association.

\section{Acknowledgements}

The authors thank the participants and their relatives and the staff of FraDySMex study.

\section{References}

1. Guerra M, Ferri CP, Sosa AL, Salas A, Gaona C, et al. (2009) Late-life depression in Peru, Mexico and Venezuela: the 10/66 populationbased study. Br J Psychiatry 195: 510-515.
2. Ortiz GG, Arias-Merino ED, Flores-Saiffe ME, Velázquez-Brizuela IE, Macías-Islas MA, et al. (2012) Prevalence of cognitive impairment and depression among a population aged over 60 years in the metropolitan area of Guadalajara, Mexico. Curr Gerontol Geriatr Res 175019.

3. Barquera S, Campos-Nonato I, Hernández-Barrera L, Pedroza A, Rivera-Dommarco JA. (2013) Prevalence of obesity in Mexican adults 2000-2012. Salud Publica Mex 55: 151-160.

4. Atlantis E, Baker M (2008) Obesity effects on depression: systematic review of epidemiological studies. Int J Obes (Lond) 32: 881-891.

5. Luppino FS, de Wit LM, Bouvy PF, Stijnen T, Cuijpers P, et al. (2010) Overweight, obesity, and depression: a systematic review and meta-analysis of longitudinal studies. Arch Gen Psychiatry 67: 220-229.

6. Carter JD, Assari S (2016) Sustained obesity and depressive symptoms over 6 years: race by gender differences in the health and Retirement Study. Front Aging Neurosci 8: 312.

7. Parra-Rodríguez L, Szlejf C, García-González Al, Malmstrom TK, Cruz-Arenas E, et al. (2016) Cross-cultural adaptation and validation of the Spanish-language version of the SARC-F to assess sarcopenia in Mexican community-dwelling older adults. J Am Med Dir Assoc 17: 1142-1146.

8. Remigio-Baker RA, Allison MA, Schreiner PJ, Carnethon MR, Nettleton JA, et al. (2015) Sex and race/ethnic disparities in the cross-sectional association between depressive symptoms and muscle mass: the Multi-ethnic Study of Atherosclerosis. BMC Psychiatry 15: 221.

9. Ishii S, Chang C, Tanaka T, Kuroda A, Tsuji T, et al. (2016) The association between Sarcopenic obesity and depressive symptoms in older Japanese adults. PLOS One 11: e0162898

10. Cho Y, Shin SY, Shin MJ (2015) Sarcopenic obesity is associated with lower indicators of psychological health and quality of life in Koreans. Nutr Res 35: 384-392.

11. Kim JH, Kim DH, Park YS (2016) Body composition, sarcopenia, and suicidal ideation in elderly Koreans: Hallym Aging Study. J Korean Med Sci 31: 604-610.

12. Kim JK, Choi SR, Choi MJ, Kim SG, Lee YK, et al. (2014) Prevalence of and factors associated with sarcopenia in elderly patients with end-stage renal disease. Clin Nutr 33: 64-8.

13. Hamer M, Batty GD, Kivimaki M (2015) Sarcopenic obesity and risk of new onset depressive symptoms in older adults: English Longitudinal Study of ageing. Int J Obes (Lond) 39: 1717-1720.

14. Byeon CH, Kang KY, Kang SH, Kim HK, Bae EJ (2016) Sarcopenia is not associated with depression in Korean adults: results from the 2010-2011 Korean national health and nutrition examination survey. Korean J Fam Med 37: 37-43.

15. Hsu YH, Liang CK, Chou MY, Liao MC, Lin YT, et al. (2014) Association of cognitive impairment, depressive symptoms and sarcopenia among healthy older men in the veterans retirement community in southern Taiwan: a cross-sectional study. Geriatr Gerontol Int 14: 102-108.

16. Rosas-Carrasco O, Cruz-Arenas E, Parra-Rodríguez L, GarcíaGonzález Al, Contreras-González LH, et al. (2016) Cross-cultural adaptation and validation of the FRAIL scale to assess frailty in Mexican adults. J Am Med Dir Assoc 17: 1094-1098.

17. Salinas-Rodríguez A, Manrique-Espinoza B, Acosta-Castillo I, TéllezRojo MM, Franco-Núñez A, et al. (2013) Validation of a cutoff for 
the Depression Scale of the Center for Epidemiologic Studies, Brief version (CESD-7). Salud publica Mex 55: 267-274.

18. (2000) Obesity: preventing and managing the global epidemic. Report of a WHO consultation. WHO Tech Rep S 894.

19. Ostrosky-Solís F, López-Arango G, Ardila A (2000) Sensitivity and specificity of the Mini-Mental State Examination in a Spanishspeaking population. Appl Neuropsychol 7: 25-31.

20. Charlson ME, Pompei P, Ales KL, MacKenzie CR (1987) A new method of classifying prognostic comorbidity in longitudinal studies: development and validation. J Chronic Dis 40: 373-383.

21. Rodríguez-Pimentel L, Garduño-Espinoza J, Padilla NE, Romo RS (2006) Concordancia en el juicio clínico al calificar la gravedad de los pacientes en los servicios de admisión. Medicina Crítica 20: 16-24.

22. Guralnik JM, Simonsick EM, Ferrucci L, Glynn RJ, Berkman LF, et al. (1994) A short physical performance battery assessing lower extremity function: association with self-reported disability and prediction of mortality and nursing home admission. J Gerontol 49: M85-M94.

23. Cabañero-Martínez MJ, Cabrero-García J, Richart-Martínez M, Muñoz-Mendoza CL (2009) The Spanish versions of the Barthel index (BI) and the Katz index (KI) of activities of daily living (ADL): a structured review. Arch Gerontol Geriatr 49: e77-e84.

24. Gnjidic D, Hilmer SN, Blyth FM, Naganathan V, Waite L, et al. (2012) Polypharmacy cutoff and outcomes: five or more medicines were used to identify community-dwelling older men at risk of different adverse outcomes. J Clin Epidemiol 65: 989-995.
25. Hinnouho GM, Singh-Manoux A, Gueguen A, Matta J, Lemogne C, et al. (2017) Metabolically healthy obesity and depressive symptoms: 16-year follow-up of the Gazel cohort study. PLOS One. 12: e0174678.

26. Goes VF, Wazlawik E, D'Orsi E, González-Chica DA (2017) Severe obesity increases the prevalence but not the incidence of depressive symptoms in the elderly-population-based cohort in Southern Brazil. Int Psychogeriatr 29: 1307-1316.

27. Qian J, Li N, Ren X. (2017) Obesity and depressive symptoms among Chinese people aged 45 and over. Sci Rep 7: 45637.

28. Lee KM, Seo MS, Shim JY, Lee YJ (2015) Body weight status misperception and its association with weight control behaviours, depressive mood and psychological distress in nulliparous normalweight young women. Ann Hum Biol 42: 528-532.

29. Heard C, Scuffham PA, Ratcliffe J, Whitty JA (2017) The association between misperceptions around weight status and quality of life in adults in Australia. Health Qual Life Outcomes 15: 53.

30. Drumond Andrade FC, Raffaelli M, Teran-Garcia M, Jerman JA, Aradillas Garcia C, et al. (2012) Up Amigos Study Group. Weight status misperception among Mexican young adults. Body Image 9: 184-188.

31. Arantxa Colchero M, Caro-Vega Y, Kaufer-Horwitz M (2014) Socioeconomic status and misperception of body mass index among Mexican adults. Salud Publica Mex 56: 251-258. 